\title{
Rikkunshito as a Therapeautic Agent for Functional Dyspepsia and its Prokinetic and Non-Prokinetic Effects
}

\author{
Kazumi Inokuchi, Tatsuhiro Masaoka* and Takanori Kanai \\ Division of Gastroenterology and Hepatology, Department of Internal Medicine, Keio University School of Medicine, Tokyo, Japan
}

OPEN ACCESS

Edited by:

Emidio Scarpellini, KU Leuven, Belgium

Reviewed by:

Jianye Yuan,

Longhua Hospital Shanghai University

of Traditional Chinese Medicine, China

Thomas Brzozowski,

Jagiellonian University Medical

College, Poland

${ }^{*}$ Correspondence:

Tatsuhiro Masaoka masaoka@keio.jp

Specialty section:

This article was submitted to Gastrointestinal and Hepatic

Pharmacology,

a section of the journal

Frontiers in Pharmacology

Received: 11 December 2020

Accepted: 10 May 2021

Published: 08 June 2021

Citation:

Inokuchi K, Masaoka T and Kanai T (2021) Rikkunshito as a Therapeautic Agent for Functional Dyspepsia and its Prokinetic and Non-Prokinetic Effects.

Front. Pharmacol. 12:640576.

doi: 10.3389/fphar.2021.640576
Prokinetics is one of the therapeutic agents for functional and motility disorders of the stomach. However, its efficacy is limited. Kampo medicine is a unique medical system that was developed in Japan. In Kampo medicine, herbal medicine is prescribed based on the patient's condition. Therefore, even for functional and motility disorders of the stomach, some herbal medicines are considered as a therapeutic option. Recently, there has been an increase in evidence for the efficacy or the mechanism of herbal medicine for functional and motility disorders of the stomach. Among these, rikkunshito is a well-studied herbal medicine that could be used as an alternative to prokinetics. In this review, we discuss the possibilities of rikkunshito for functional dyspepsia with its prokinetic and non-prokinetic effects and provide an overview of their current use with a focus on their therapeutic mechanism.

Keywords: functional and motility disorders of the stomach, non-prokinetic treatment, rikkunshito, Kampo, functional dyspepsia

\section{INTRODUCTION}

Kampo is a traditional herbal medicine that was introduced from China to Japan. After that, Kampo medicine has been uniquely developed in Japan. Research on Kampo medicine has thus been studied mainly in East Asia and has not yet garnered worldwide study. This way of evaluating patients is different from Western medicine. The most characteristic aspect of Kampo medicine lies in how it is used to evaluate patients. It evaluates the whole body condition, called the "pattern" and "qi, blood, and fluid", not each organ. Based on the patient's condition, herbal medicine is prescribed in Kampo medicine. Therefore, its application is broad and can include patients with Functional Dyspepsia (FD). Some herbal medicines are prescribed to patients with FD, and, of these, rikkunshito is a well-studied herbal medicine. This review focuses on rikkunshito and discusses its potential as an alternative therapeutic option for FD besides traditional treatments.

\section{PATHOPHYSIOLOGY AND TREATMENT OF FD}

Functional gastrointestinal disorders (FGIDs) are characterized by subjective abdominal symptoms and lack of organic disease. FGIDs are defined by the Rome IV criteria, which were revised in 2016 (Drossman and Hasler, 2016). Functional dyspepsia (FD) is one of the FGIDs of gastroduodenal lesions. In the Rome IV criteria, symptoms of FD are bothersome postprandial fullness, early satiation, epigastric pain, and epigastric burning occurring more than 6 months prior to diagnosis and persisting for more than 3 months. FD is sub-classified as post-prandial distress syndrome (PDS) or epigastric pain syndrome (EPS), and these sub-classifications can overlap. 
The pathophysiology of FD is heterogeneous, and it involves various pathological conditions, such as dysmotility, visceral hypersensitivity, low-grade inflammation, dysregulation of the gut-brain axis, and psychological factors. Recently, the involvement of impaired duodenal mucosal integrity has been suggested (Vanheel et al., 2014; Wauters et al., 2020), although the details of the mechanism have not been fully elucidated.

Because of the heterogeneity in pathophysiology, the basic treatment of FD has not yet been established. When FD is diagnosed, the presence of a Helicobacter pylori infection should be checked for and, if present, treated. If an infection is absent or symptoms do not improve after treatment, proton pump inhibitors (PPI) and $\mathrm{H}_{2}$ receptor blockers, or prokinetics such as acotiamide, domperidone, mosapride, and itopride, should be used. If no improvement is observed, additional antidepressants are recommended (Moayyedi et al., 2017).

The usefulness of PPI is constantly discussed. Some reports indicated that a 2-8-week dosing is more useful than placebo (Pinto-Sanchez et al., 2017), while others suggested that it is useful when complicated by gastroesophageal reflux disease (GERD) and is ineffective on FD itself (Wong et al., 2002; Pinto-Sanchez et al., 2017; Tack and Camilleri, 2018), and the efficacy of PPI is thus controversial. Although PPI are generally considered safe and well tolerated, they are associated with a risk of developing a Clostridium difficile infection, pneumonia, fractures, and acute interstitial nephritis with long-term administration (Wilhelm et al., 2013).

Although the effectiveness of prokinetics for FD was analyzed in the meta-analysis, there is a variation in the report (Moayyedi et al., 2017), and the evidence level is not high. Thus, a highquality randomized controlled trial (RCT) is desirable (Pittayanon et al., 2018).

FD is not a life-threatening disease; however, it remarkably burdens the quality of life. Therefore, establishing effective therapy is urgently needed.

\section{WHAT IS RIKKUNSHITO?}

Rikkunshito is an herbal medicine that is widely used for improving gastrointestinal symptoms, such as FD, mainly in Asian countries. In China, it is called Liu-Jun-Zi Tang, and it is a Chinese herbal formula originally described in the Chinese classic medical book.

In Kampo medicine, when prescribing herbal medicine, the condition of patients is often assessed using the labels "pattern" and "qi, blood, and fluid." This concept markedly differs from that of Western medicine. In brief, the "pattern" is the evaluation of physical fitness and resistance to illness, and "qi, blood, and fluid" considers the causes of the disorder in these three parts. One way of dividing the "pattern" is "excess or deficiency." Individuals who are thin, delicate, and prone to diarrhea and chilling and those with weak gastrointestinal function a low stamina are classified as having a deficiency. Conversely, those who have sufficient energy and muscular strength are classified as having an excess pattern. Rikkunshito is prescribed mainly for patients with a deficiency pattern. When translating rikkunshito directly to English, it would be translated to "soup of six nobles." Rikkunshito is composed of the following eight herbal medicines: extracts of Atractylodes lancea Rhizome, Ginseng, Pinellia tuber, Poria sclerotium, Jujube, Citrus unshiu Peel, Glycyrrhiza, and Ginger. Among these, the extracts of the Atractylodes lancea Rhizome, Ginseng, Poria sclerotium, Pinellia tuber, Citrus unshiu Peel, and Glycyrrhiza were imagined as the six nobles (Yagi et al., 2004). It has been clarified that the ingredients of rikkunshito include $\beta$-eudesmol derived from the Atractylodes lancea Rhizome, ginsenosides derived from the Ginseng, hesperidin derived from the Citrus unshiu Peel, glycyrrhizin derived from the Glycyrrhiza, and shogaol derived from the ginger. Rikkunshito is widely used for upper gastrointestinal disorders in Japan. As side effects, hepatic dysfunction or pseudoaldosteronism owing to glycyrrhizin contained in Glycyrrhiza are known.

\section{Clinical Effect of Rikkunshito on FD}

In 1993, Tatsuta et al. (Tatsuta and Iishi, 1993) divided 42 patients with FD into the rikkunshito group $(N=22)$ and the placebo group $(N=20)$ and compared gastric emptying using the acetaminophen absorption method. They reported that gastrointestinal symptoms significantly improved and gastric emptying was enhanced in the rikkunshito group after 7 days of medication. In 2014, Suzuki et al. performed a double-blind RCT, dividing FD patients into a rikkunshito group and a placebo group where patients received 8 weeks of medication. Improvement of epigastric pain $(p=0.04)$ and postprandial fullness $(p=0.06)$ were observed in the group treated with rikkunshito, and this suggested that the efficacy may be lower in H. pylori-uninfected individuals (Suzuki et al., 2014). In a post hoc analysis (Togawa et al., 2016), it has been reported that a lower level of ghrelin in blood was an independent factor supporting the effectiveness of rikkunshito in FD patients without $H$. pylori infection and that it was more therapeutically effective in patients with $H$. pylori infection in the absence of alcohol consumption. Another double-blind, RCT was conducted in Japan in which 192 FD patients who met the Rome III criteria were enrolled (Tominaga et al., 2018). After a 2-week single-blind placebo period, patients were divided into the rikkunshito group $(N=64)$ and the placebo group $(N=61)$ for 8 weeks. The authors showed significant improvement in psychiatric symptoms and upper gastrointestinal symptoms in the rikkunshito group (Tominaga et al., 2018). However, a systematic review and a meta-analysis review have suggested that further studies are needed for supporting the usefulness of rikkunshito (Hoshino et al., 2019). Mausy et al. examined the effect of rikkunshito on symptoms and gastric motility in European FD-PDS patients using a randomized, placebo-controlled, crossover study. Rikkunshito did not alter gastric motility. Treatment with rikkunshito improved upper gastrointestinal symptoms in FD patients, but similarly, high placebo effects were observed (Masuy et al., 2020). A meta-analysis has reported that the combination of Western medication and rikkunshito was more helpful than the use of Western medication alone (Ko 
et al., 2020). Further accumulation of evidence for the clinical effect of rikkunshito on FD is demanded.

\section{MECHANISM OF ACTION OF RIKKUNSHITO ON SENSORY-MOTOR FUNCTION IN ANIMALS VIA THE NON-GHRELIN MEDIATED PATHWAY}

When the effects of metoclopramide, trimebutine, cisapride, and rikkunshito were examined on the gastric adaptive relaxation in guinea pig stomachs, only rikkunshito induced adaptive relaxation of the stomach and improvement of the gastric volume. Oral administration of $\mathrm{N}(\mathrm{G})$-nitro-L-arginine (L-NNA), a nitic oxide (NO) synthetic inhibitor to the stomach, inhibited gastric adaptive relaxation. Rikkunshito, but not gastroprokinetics, overcame the effect of the NG-nitro-Larginine. This result suggests the action of rikkunshito via the NO-mediated pathway (Hayakawa et al., 1999).

Serotonin (5-hydroxytryptamine (5-HT)) is synthesized from tryptophan, and $90 \%$ of 5-HT exists in the gastrointestinal tract. 5-HT regulates the sensitization, motility, and secretion of the gastrointestinal tract. 5-HT receptors are classified into seven families and 14 subtypes. Among these, $5-\mathrm{HT}_{3}$ and $5-\mathrm{HT}_{4}$ receptors are involved in the regulation of sensation and motor function in the gastrointestinal tract (Grider et al., 1998; Taniyama et al., 2000), and 5- $\mathrm{HT}_{2 \mathrm{C}}$ is involved in eating behavior.

The efficacy of rikkunshito for cisplatin-induced anorexia in rats has been reported. Administration of 5-HT and dopamineinduced delayed gastric emptying in rats. Administration of a 5$\mathrm{HT}_{3}$ receptor antagonist, ondansetron, and rikkunshito did not improve the delayed gastric emptying induced by dopamine but ameliorated the delayed gastric emptying induced by $5-\mathrm{HT}$. Furthermore, their effect was abolished by atropine administration. Therefore, the $5-\mathrm{HT}_{3}$ receptor-mediated pathway has been suggested as a mechanism of action of rikkunshito (Tominaga et al., 2011). Additionally, several flavonoids contained in rikkunshito cross the blood-brain barrier. The action of rikkunshito for anorexia may be mediated via $5-\mathrm{HT}_{2 \mathrm{C}}$ receptors in the central nervous system (Arai et al., 2013). Moreover, association between plasma ACTH and cortisol levels under stress and rikkunshito is also reported (Naito et al., 2003).

Regarding gastric emptying, the effect of rikkunshito is mainly studied with rats. Improvement of delayed gastric emptying via the NO-mediated pathway is reported. In this study, as ingredients in rikkunshito, roles of Hesperidin in Citrus unshu Peel and L-Arginine in Ginseng, Pinella ternate and Atractylodes lancea Rhizome are suggested (Kido et al., 2005). Improvement of delayed gastric emptying via antagonistic action of the 5-HT(3) receptor is suggested (Sadakane et al., 2011). Moreover, potentiation of phase IIIlike contractions by rikkunshito is reported. In this study, as ingredients in rikkunshito, roles of Hesperidin in Citrus unshu Peel, and L-Arginine in Ginseng, Pinella ternate and
Atractylodin in Atractylodes lancea Rhizome are suggested (Nahata et al., 2018). Regarding gastric adaptive relaxation or gastric accommodation, the effect of rikkunshito is mainly studied with guinea pigs. Potentiation of gastric adaptive relaxation is reported (Hayakawa et al., 1999). Moreover, suppression of impairment of gastric accommodation via the NO- and 5-HT(2B) receptor-mediated pathway is reported (Miwa et al., 2016). These findings are summarized in Table 1.

In 2020, Zhao et al. reported that rikkunshito significantly attenuated visceral hypersensitivity in FD model rats, and the overexpression of EC cells, 5HT, TPH1, PAX4, and 5HT3R in the duodenum of FD model rats was also reduced by rikkunshito decoction (Zhao et al., 2020).

\section{MECHANISM OF ACTION OF RIKKUNSHITO VIA THE GHRELIN-MEDIATED PATHWAY}

Ghrelin is a 28-amino-acid peptide identified as a ligand for the growth hormone secretagogue receptor (GHS-R) in 1999. Ghrelin is mainly secreted by gastric endocrine X/A-like cells (Kojima et al., 1999). Secreted ghrelin enhances food intake through the activation of food intake stimulating neuropeptide Y (NPY)/agouti-related protein (AgRP) neurons and the inhibition of anorexigenic proopiomelanocortin (POMC) neurons. These neurons exist in the arcuate nucleus of the hypothalamus (Kageyama et al., 2010; Andrews, 2011). Ghrelin regulates growth hormone secretion and feeding, and the blood level of ghrelin is negatively correlated with the body mass index in healthy individuals and uncomplicated type 2 diabetes mellitus patients; it has been reported to be lower in obese patients and higher in lean patients with cancer, chronic heart failure, and anorexia nervosa (Ueno et al., 2007). Administration of a selective serotonin uptake inhibitor containing fenfluramine reduced blood ghrelin levels and gastrointestinal peristalsis in rats. Oral administration of rikkunshito improved blood ghrelin levels and dietary intake (Fujitsuka et al., 2009). In clinical studies in humans, the administration of rikkunshito increases blood ghrelin levels in healthy participants and in patients after gastrectomy (Fujitsuka and Uezono, 2014). Rikkunshito may ameliorate anorexia by promoting the secretion of ghrelin. In 2008, Takeda et al. firstly reported the relationship between rikkunshito and ghrelin. They reported oral administration of rikkunshito suppressed the cisplatin-induced decrease in plasma acylated-ghrelin levels. (Takeda et al., 2008). Since then, a variety of rikkunshito's ghrelin-mediated mechanisms have become apparent. Thirtytwo ingredients were considered as typical in rikkunshito. In total, 18 or 21 ingredients from plasma or urine were detected after administration of rikkunshito in healthy volunteer study (Kitagawa et al., 2015). Among these, 9 ingredients were elucidated their mechanism as ghrelin mediated therapeutic effect of rikkunshito by in vivo and in vitro study. Pachymic acid in Poria sclerotium and 10-gingerol in Ginger inhibit 
TABLE 1 | List of in vivo studies that examine the efficacy of rikkunshito on motor patterns.

\begin{tabular}{|c|c|c|c|c|}
\hline Effect & Ingredient & Mechanism & Utilized animals & References \\
\hline Improvement of delayed gastric emptying & Hesperidin, L-Arginine & NO mediated action & Rats & Kido et al. (2005) \\
\hline Improvement of delayed gastric emptying & & Antagonistic action of the $5-\mathrm{HT}(3) \mathrm{R}$ & Rats & Tominaga et al. (2011) \\
\hline Potentiate phase III-like contractions & Atractylodin & & Rats & Nahata et al. (2018) \\
\hline Potentiate gastric adaptive relaxation & & & Guinea pigs & Hayakawa et al. (1999) \\
\hline Suppress impairment of gastric accommodation & & $\mathrm{NO}$ and $5-\mathrm{HT}(2 \mathrm{~B}) \mathrm{R}$ mediated pathway & Guinea pigs & Miwa et al. (2016) \\
\hline
\end{tabular}

NO, nitric oxide; 5-hydroxytryptamine 3 receptor, 5-HT(3) $R$; 5-hydroxytryptamine $2 B$ receptor, 5-HT(2B)R.

\begin{tabular}{|c|c|c|c|c|}
\hline Herb & Ingredient & Target & Role & References \\
\hline Poria sclerotium & Pachymic acid & & Inhibit ghrelin degration & Sadakane et al. (2011) \\
\hline \multirow[t]{4}{*}{ Citrus unshu Peel } & Heptamethoxyflavone & PDEIII & Improve ghrelin resistance & Takeda et al. (2010) \\
\hline & Nobiletin & PDEIII & Improve ghrelin resistance & Takeda et al. (2010) \\
\hline & Isoliquiritigenin & PDEIII & Improve ghrelin resistance & Takeda et al. (2010) \\
\hline & Hesperidin & 5-HT(2C)R & Promote ghrelin secretion & Nahata et al. (2013) \\
\hline \multirow[t]{2}{*}{ Ginger } & 8-Gingerol & 5-HT(2C)R & Promote ghrelin sectertion & Nahata et al. (2013) \\
\hline & 10-Gingerol & & Inhibit ghrelin degration & Sadakane et al. (2011) \\
\hline Glycyrrhiza & Isoliquiritigenin & 5-HT(2B)R & Promote ghrelin secretion & Yamada et al. (2013) \\
\hline Atractylodes lancea Rhizome & Atractylodin & & Promote ghrelin secretion & Fujitsuka et al. (2011) \\
\hline
\end{tabular}

PDE III, phosphodiesterase III; 5-hydroxytryptamine $2 \mathrm{C}$ receptor, 5-HT(2C)R, 5-hydroxytryptamine $2 B$ receptor, 5-HT(2B)R.

ghrelin diacylation by inhibiting ghrelin-deacylating enzymes (Sadakane et al., 2011). Heptamethoxyflavone, Nobiletin, and Isoliquiritigenin in Citrus unshu Peel improve ghrelin resistance via central phosphodiesterase III inhibition (Takeda et al., 2010). Atractylodin in Atractylodes lancea Rhizome, Hesperidin in Citrus unshu Peel, 8-gingerol in Ginger, and Isoliquiritigenin in Glycyrrhiza promote ghrelin secretion (Fujitsuka et al., 2011; Nahata et al., 2013; Yamada et al., 2013). Particularly, Hesperidin and 8-gingerol promote ghrelin secretion by central 5-HT2C receptor antagonism, and Isoliquiritigenin promotes ghrelin secretion via peripheral 5-HT2B receptor antagonism. The effects of the above ingredients of rikkunshito are summarized in Table 2.

Most of the circulating ghrelin is synthesized and secreted by $\mathrm{X} / \mathrm{A}$-like cells in the stomach. Morevoer, neurons in the hypothalamus also express ghrelin (Castañeda et al., 2010). As mentioned above, rikkunshito has both of peripheral and central effect for promoting ghrelin secretion. However, whether the central or peripheral effect of rikkunshito is more important for promoting ghrelin secretion is not clarified yet.

The plasma levels of ghrelin decrease with increasing gastric mucosal atrophy (Suzuki et al., 2004). In the randomized clinical trial of rikkunshito for the treatment of functional dyspepsia, rikkunshito was relatively more effective among Helicobacter pylori-infected participants (rikkunshito: $40.0 \%$ vs. placebo: $20.5 \%, p=0.07$ ) and seemed less effective among $H$. pylori-uninfected participants (rikkunshito: $29.3 \%$ vs. placebo: $25.6 \%, p=$ 0.72). Among H. pylori-positive individuals, acyl ghrelin levels were improved just in rikkunshito group (Suzuki et al., 2014). From these findings, there is a possibility that rikkunshito returns the ghrelin level to normal when the ghrelin secretion is below the normal level.

A randomised double-blind cross-over study of healthy volunteers showed an increase in the appetite and energy consumption in the free-choice buffet (Wren et al., 2001). Therefore, long term administration of ghrelin may have a risk for evoking obesity. However, to the best of our knowledge, a risk of obesity has not yet been linked to rikkunshito administration.

\section{DISCUSSION}

As mentioned above, in an in vivo study, the mechanisms of action of rikkunshito, such as promoting ghrelin secretion, mediating gastric adaptive relaxation, and stimulating gastric emptying, have been elucidated (Kido et al., 2005; Arai et al., 2013; Fujitsuka and Uezono, 2014). The effect of rikkunshito on gastric motility in humans has been studied with various modalities. Kusunoki et al. measured the cross-sectional area of the proximal part of the stomach using abdominal ultrasound and calculated the gastric emptying rate, motility index, and reflux index before and after administration of rikkunshito in patients with FD. However, there was no improvement in symptoms; all measurements except the reflux index significantly increased after the administration of rikkunshito (Kusunoki et al., 2010). Shiratori et al. used a barostat for measuring the volume of the stomach before and after the administration of rikkunshito in nine healthy volunteers and reported that the 


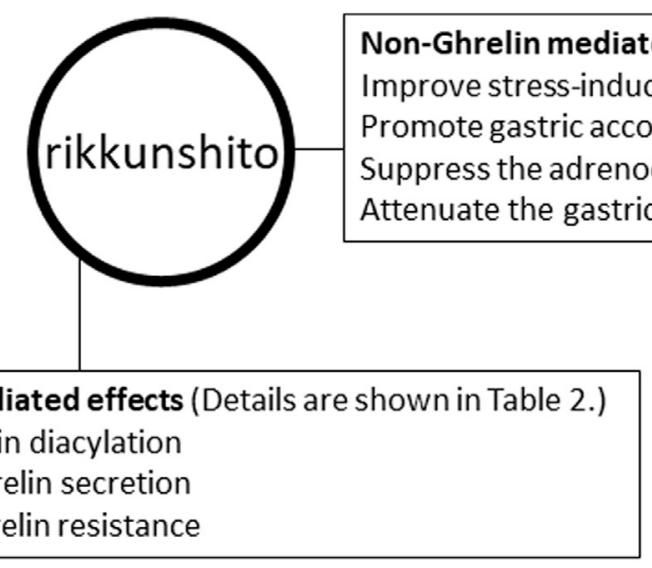

Ghrelin mediated effects (D
Inhibit ghrelin diacylation
Promote ghrelin secretion
Improve ghrelin resistance

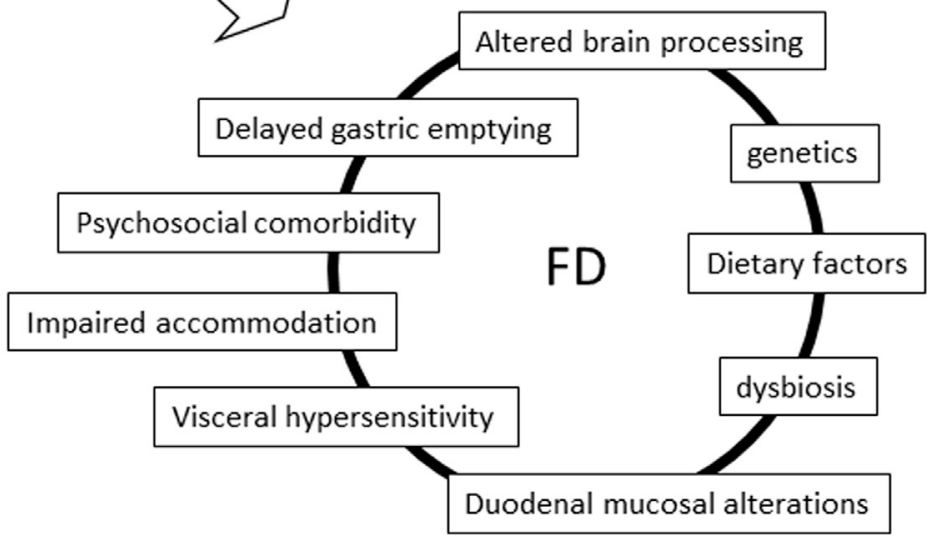

FIGURE 1 | Multiple effects of rikkunshito and multiple pathophysiologies of functional dyspepsia.

volume of the stomach increased significantly after the administration, with improvement in perceived stress (Shiratori et al., 2011). Mausy et al. examined the effect of rikkunshito on intra-gastric pressure with high-resolution manometry before and after treatment with rikkunshito; however, rikkunshito did not alter gastric motility (Masuy et al., 2020). These differences might be caused by the characteristics of each modality. Regarding the effect of rikkunshito, in vivo studies were ahead of clinical studies. Compared with the results of the aforementioned in vivo studies, human studies did not entirely reproduce results of in vivo studies. Further research on the effects of rikkunshito on the human stomach and its association with symptomatic improvement is needed.

As described in the Introduction section, Kampo medicine, in contrast to Western medicine, a suitable drug for the patient is prescribed based on the assessment of the patient's pattern in addition to the symptoms. Furthermore, herbal medicine contains a large number of active ingredients; the effects of herbal medicine come from intertwined ingredients and have a complex mechanism of action. They can act on the whole body rather than locally. Kampo medicine may not be very popular in Western countries because of the differences in the way of choosing herbal medicine. Herbal medicine used in Kampo medicine includes multiple herbals, and each herb includes many chemicals. Therefore, herbal medicine has various mechanisms, whereas a western drug is generally composed of a single chemical with a single mechanism of action (Yu et al., 2006).

Moreover, because many of the previous studies on Kampo medicine conducted in the 1980s-1990s have been published in Japanese, these pioneering studies have not been referred to in English literature; they have not been indexed in PubMed. This inaccessibility of studies may be one of the reasons for the difficulty in sharing the results globally (Oka et al., 2014). There is also a possibility that unexpected drug interactions may emerge in combination with Western medicines. Some side effects and components of herbal medicine, such as pseudoaldosteronism and glycyrrhizin in Glycyrrhiza, have been identified. Though it is not so for all cases, some drug interaction between herbal medicine and Western drugs could be solved through cautious use; therefore, the possibility of safe usage of Kampo in Europe is suggested (Homma, 2016).

As mentioned above, Rikkunshito can exert prokinetic activity and other effects, such as visceral hypersensitivity alleviation. There is no high-quality and sufficient evidence on the effectiveness of rikkunshito on FD. In contrast, it may be of 
sufficient value to attempt approaches applying Kampo medicine for complex and poorly understood pathologies, such as FD (Figure 1). In the future, we hope that Kampo, including rikkunshito, will become popular worldwide, and studies to elucidate the disease state of FD and the efficacy of Kampo will be actively performed.

\section{AUTHOR CONTRIBUTIONS}

KI wrote the initial draft of the manuscript and revised the manuscript. TM designed this mini review and revised the

\section{REFERENCES}

Andrews, Z. B. (2011). Central Mechanisms Involved in the Orexigenic Actions of Ghrelin. Peptides 32, 2248-2255. doi:10.1016/j.peptides.2011.05.014

Arai, T., Maejima, Y., Muroya, S., and Yada, T. (2013). Rikkunshito and Isoliquiritigenin Counteract 5-HT-Induced 2C Receptor-Mediated Activation of Pro-opiomelanocortin Neurons in the Hypothalamic Arcuate Nucleus. Neuropeptides 47, 225-230. doi:10.1016/j.npep.2013.05.004

Castañeda, T. R., Tong, J., Datta, R., Culler, M., and Tschöp, M. H. (2010). Ghrelin in the Regulation of Body Weight and Metabolism. Front. Neuroendocrinology 31, 44-60. doi:10.1016/j.yfrne.2009.10.008

Drossman, D. A., and Hasler, W. L. (2016). Rome IV-Functional GI Disorders: Disorders of Gut-Brain Interaction. Gastroenterology 150, 1257-1261. doi:10. 1053/j.gastro.2016.03.035

Fujitsuka, N., Asakawa, A., Hayashi, M., Sameshima, M., Amitani, H., Kojima, S., et al. (2009). Selective Serotonin Reuptake Inhibitors Modify Physiological Gastrointestinal Motor Activities via 5-HT2c Receptor and Acyl Ghrelin. Biol. Psychiatry 65, 748-759. doi:10.1016/j.biopsych.2008.10.031

Fujitsuka, N., Asakawa, A., Uezono, Y., Minami, K., Yamaguchi, T., Niijima, A., et al. (2011). Potentiation of Ghrelin Signaling Attenuates Cancer Anorexia-Cachexia and Prolongs Survival. Transl Psychiatry 1, e23. doi:10.1038/tp.2011.25

Fujitsuka, N., and Uezono, Y. (2014). Rikkunshito, a Ghrelin Potentiator, Ameliorates Anorexiaâ€"cachexia Syndrome. Front. Pharmacol. 5, 271. doi:10.3389/fphar.2014.00271

Grider, J. R., Foxx-Orenstein, A. E., and Jin, J.-G. (1998). 5-Hydroxytryptamine4 Receptor Agonists Initiate the Peristaltic Reflex in Human, Rat, and guinea Pig Intestine. Gastroenterology 115, 370-380. doi:10.1016/s0016-5085(98)70203-3

Hayakawa, T., Arakawa, T., Kase, Y., Akiyama, S., Ishige, A., Takeda, S., et al. (1999). Liu-Jun-Zi-Tang, a Kampo Medicine, Promotes Adaptive Relaxation in Isolated guinea Pig Stomachs. Drugs Exp. Clin. Res. 25, 211-218.

Homma, M. (2016). Education Program of Kampo-Medicine for Undergraduates in Preparation for Clinical Setting. Yakugaku. Zasshi. 136, 417-422. doi:10. 1248/yakushi.15-00232-4

Hoshino, N., Nishizaki, D., Hida, K., Obama, K., and Sakai, Y. (2019). Rikkunshito for Upper Gastrointestinal Symptoms: A Systematic Review and Meta-Analysis. Complement. Therapies Med. 42, 255-263. doi:10.1016/j.ctim.2018.11.025

Kageyama, H., Takenoya, F., Shiba, K., and Shioda, S. (2010). Neuronal Circuits Involving Ghrelin in the Hypothalamus-Mediated Regulation of Feeding. Neuropeptides 44, 133-138. doi:10.1016/j.npep.2009.11.010

Kido, T., Nakai, Y., Kase, Y., Sakakibara, I., Nomura, M., Takeda, S., et al. (2005). Effects of Rikkunshi-To, a Traditional Japanese Medicine, on the Delay of Gastric Emptying Induced by NG-Nitro-L-arginine. J. Pharmacol. Sci. 98, 161-167. doi:10.1254/jphs.fpj04056x

Kitagawa, H., Munekage, M., Matsumoto, T., Sadakane, C., Fukutake, M., Aoki, K., et al. (2015). Pharmacokinetic Profiles of Active Ingredients and its Metabolites Derived from Rikkunshito, a Ghrelin Enhancer, in Healthy Japanese Volunteers: A Cross-Over, Randomized Study. PLoS One 10 (7), e0133159. doi:10.1371/journal.pone.0133159

Ko, S. J., Park, J., Kim, M. j., Kim, J., and Park, J. W. (2020). Effects of the Herbal Medicine Rikkunshito, for Functional Dyspepsia: A Systematic Review and Meta-analysis. J. Gastroenterol. Hepatol. 36, 64-74. doi:10.1111/jgh.15208 manuscript. TK critically reviewed the manuscript, and all authors approved the final version of the manuscript and agreed to be accountable for all aspects of the work by ensuring that questions related to the accuracy or integrity of any part of the work are appropriately investigated and resolved.

\section{ACKNOWLEDGMENTS}

Authors acknowledge Editage (www.editage.jp) for English language editing.

Kojima, M., Hosoda, H., Date, Y., Nakazato, M., Matsuo, H., and Kangawa, K. (1999). Ghrelin Is a Growth-Hormone-Releasing Acylated Peptide from Stomach. Nature 402, 656-660. doi:10.1038/45230

Kusunoki, H., Haruma, K., Hata, J., Ishii, M., Kamada, T., Yamashita, N., et al. (2010). Efficacy of Rikkunshito, a Traditional Japanese Medicine (Kampo), in Treating Functional Dyspepsia. Intern. Med. 49, 2195-2202. doi:10.2169/ internalmedicine. 49.3803

Masuy, I., Carbone, F., Holvoet, L., Vandenberghe, A., Vanuytsel, T., and Tack, J. (2020). The Effect of Rikkunshito on Gastrointestinal Symptoms and Gastric Motor Function: the First Study in a Belgian Functional Dyspepsia Population. Neurogastroenterology Motil. 32, e13739. doi:10.1111/nmo.13739

Miwa, H., Koseki, J., Oshima, T., Hattori, T., Kase, Y., Kondo, T., et al. (2016). Impairment of Gastric Accommodation Induced by Water-avoidance Stress Is Mediated by 5- HT 2B Receptors. Neurogastroenterol. Motil. 28, 765-778. doi:10.1111/nmo.12775

Moayyedi, P. M., Lacy, B. E., Andrews, C. N., Enns, R. A., Howden, C. W., and Vakil, N. (2017). ACG and CAG Clinical Guideline: Management of Dyspepsia. Am. J. Gastroenterol. 112, 988-1013. doi:10.1038/ajg.2017.154

Nahata, M., Mizuhara, Y., Sadakane, C., Watanabe, J., Fujitsuka, N., and Hattori, T. (2018). Influence of Food on the Gastric Motor Effect of the Kampo Medicine Rikkunshito in Rat. Neurogastroenterol Motil. 30, e13177. doi:10.1111/nmo.13177

Nahata, M., Muto, S., Nakagawa, K., Ohnishi, S., Sadakane, C., Saegusa, Y., et al. (2013). Serotonin 2C Receptor Antagonism Ameliorates novelty-induced Hypophagia in Aged Mice. Psychoneuroendocrinology 38, 2051-2064. doi:10. 1016/j.psyneuen.2013.03.014

Naito, T., Itoh, H., and Takeyama, M. (2003). Some Gastrointestinal Function Regulatory Kampo Medicines Have Modulatory Effects on Human Plasma Adrenocorticotropic Hormone and Cortisol Levels with Continual Stress Exposure. Biol. Pharm. Bull. 26, 101-104. doi:10.1248/bpb.26.101

Oka, T., Okumi, H., Nishida, S., Ito, T., Morikiyo, S., Kimura, Y., et al. (2014). Effects of Kampo on Functional Gastrointestinal Disorders. Biopsychosocial Med. 8, 5. doi:10.1186/1751-0759-8-5

Pinto-Sanchez, M. I., Yuan, Y., Bercik, P., and Moayyedi, P. (2017). Proton Pump Inhibitors for Functional Dyspepsia. Cochrane. Database. Syst. Rev. 3, Cd011194. doi:10.1002/14651858.CD011194.pub2

Pittayanon, R., Yuan, Y., Bollegala, N. P., Khanna, R., Leontiadis, G. I., and Moayyedi, P. (2018). Prokinetics for Functional Dyspepsia. Cochrane. Database. Syst. Rev. 10, Cd009431. doi:10.1002/14651858.CD009431. pub3

Sadakane, C., Muto, S., Nakagawa, K., Ohnishi, S., Saegusa, Y., Nahata, M., et al. (2011). 10-Gingerol, a Component of Rikkunshito, Improves Cisplatin-Induced Anorexia by Inhibiting Acylated Ghrelin Degradation. Biochem. Biophysical Res. Commun. 412, 506-511. doi:10.1016/j.bbrc.2011.08.002

Shiratori, M., Shoji, T., Kanazawa, M., Hongo, M., and Fukudo, S. (2011). Effect of Rikkunshito on Gastric Sensorimotor Function under Distention. Neurogastroenterol. Motil. 23,-326. doi:10.1111/j.1365-2982.2010.01648.x

Suzuki, H., Masaoka, T., Hosoda, H., Nomura, S., Ohara, T., Kangawa, K., et al. (2004). Plasma Ghrelin Concentration Correlates with the Levels of Serum Pepsinogen I and Pepsinogen I/II Ratio-Aa Possible Novel and Non-invasive Marker for Gastric Atrophy. Hepatogastroenterology 51, 1249-1254.

Suzuki, H., Matsuzaki, J., Fukushima, Y., Suzaki, F., Kasugai, K., Nishizawa, T., et al. (2014). Randomized Clinical Trial: Rikkunshito in the Treatment of Functional 
Dyspepsia-A Multicenter, Double-Blind, Randomized, Placebo-Controlled Study. Neurogastroenterol. Motil. 26, 950-961. doi:10.1111/nmo.12348

Tack, J., and Camilleri, M. (2018). New Developments in the Treatment of Gastroparesis and Functional Dyspepsia. Curr. Opin. Pharmacol. 43, 111-117. doi:10.1016/j.coph.2018.08.015

Takeda, H., Muto, S., Hattori, T., Sadakane, C., Tsuchiya, K., Katsurada, T., et al. (2010). Rikkunshito Ameliorates the Aging-Associated Decrease in Ghrelin Receptor Reactivity via Phosphodiesterase III Inhibition. Endocrinology 151, 244-252. doi:10.1210/en.2009-0633

Takeda, H., Sadakane, C., Hattori, T., Katsurada, T., Ohkawara, T., Nagai, K., et al. (2008). Rikkunshito, an Herbal Medicine, Suppresses Cisplatin-Induced Anorexia in Rats via 5-HT2 Receptor Antagonism. Gastroenterology 134, 2004-2013. doi:10.1053/j.gastro.2008.02.078

Taniyama, K., Makimoto, N., Furuichi, A., Sakurai-Yamashita, Y., Nagase, Y., Kaibara, M., et al. (2000). Functions of Peripheral 5-hydroxytryptamine Receptors, Especially 5-hydroxytryptamine 4 Receptor, in Gastrointestinal Motility. J. Gastroenterol. 35, 575-582. doi:10.1007/s005350070056

Tatsuta, M., and Iishi, H. (1993). Effect of Treatment with Liu-Jun-Zi-Tang (TJ43) on Gastric Emptying and Gastrointestinal Symptoms in Dyspeptic Patients. Aliment. Pharmacol. Ther. 7, 459-462. doi:10.1111/j.1365-2036. 1993.tb00120.x

Togawa, K., Matsuzaki, J., Kobayakawa, M., Fukushima, Y., Suzaki, F., Kasugai, K., et al. (2016). Association of Baseline Plasma Des-Acyl Ghrelin Level with the Response to Rikkunshito in Patients with Functional Dyspepsia. J. Gastroenterol. Hepatol. 31, 334-341. doi:10.1111/jgh.13074

Tominaga, K., Kido, T., Ochi, M., Sadakane, C., Mase, A., Okazaki, H., et al. (2011). The Traditional Japanese Medicine Rikkunshito Promotes Gastric Emptying via the Antagonistic Action of the 5-HT3Receptor Pathway in Rats. Evidence-Based Complement. Altern. Med. 2011, 1-8. doi:10.1093/ ecam $/$ nep 173

Tominaga, K., Sakata, Y., Kusunoki, H., Odaka, T., Sakurai, K., Kawamura, O., et al. (2018). Rikkunshito Simultaneously Improves Dyspepsia Correlated with Anxiety in Patients with Functional Dyspepsia: a Randomized Clinical Trial (The DREAM Study). Neurogastroenterol. Motil. 30, e13319, doi:10.1111/nmo. 13319

Ueno, H., Shiiya, T., Mizuta, M., Mondal, M., and Nakazato, M. (2007). Plasma Ghrelin Concentrations in Different Clinical Stages of Diabetic Complications and Glycemic Control in Japanese Diabetics. Endocr. J. 54, 895-902. doi:10. 1507/endocr..k07-007

Vanheel, H., Vicario, M., Vanuytsel, T., Van Oudenhove, L., Martinez, C., Keita, Å. V., et al. (2014). Impaired Duodenal Mucosal Integrity and Low-Grade Inflammation in Functional Dyspepsia. Gut 63, 262-271. doi:10.1136/gutjnl2012-303857
Wauters, L., Talley, N. J., Walker, M. M., Tack, J., and Vanuytsel, T. (2020). Novel Concepts in the Pathophysiology and Treatment of Functional Dyspepsia. Gut 69, 591-600. doi:10.1136/gutjnl-2019-318536

Wilhelm, S. M., Rjater, R. G., and Kale-Pradhan, P. B. (2013). Perils and Pitfalls of Long-Term Effects of Proton Pump Inhibitors. Expert Rev. Clin. Pharmacol. 6, 443-451. doi:10.1586/17512433.2013.811206

Wong, W. M., Wong, B. C., Hung, W. K., Yee, Y. K., Yip, A. W., Szeto, M. L., et al. (2002). Double Blind, Randomised, Placebo Controlled Study of Four Weeks of Lansoprazole for the Treatment of Functional Dyspepsia in Chinese Patients. Gut 51, 502-506. doi:10.1136/gut.51.4.502

Wren, A. M., Seal, L. J., Cohen, M. A., Brynes, A. E., Frost, G. S., Murphy, K. G., et al. (2001). Ghrelin Enhances Appetite and Increases Food Intake in Humans. J. Clin. Endocrinol. Metab. 86, 5992. doi:10.1210/jcem.86.12.8111

Yagi, M., Homma, S., Kubota, M., Iinuma, Y., Kanada, S., Kinoshita, Y., et al. (2004). The Herbal Medicine Rikkunshi-To Stimulates and Coordinates the Gastric Myoelectric Activity in post-operative Dyspeptic Children after Gastrointestinal Surgery. Pediatr. Surg. Int. 19, 760-765. doi:10.1007/ s00383-003-1053-y

Yamada, C., Saegusa, Y., Nakagawa, K., Ohnishi, S., Muto, S., Nahata, M., et al. (2013). Rikkunshito, a Japanese Kampo Medicine, Ameliorates Decreased Feeding Behavior via Ghrelin and Serotonin 2B Receptor Signaling in a Novelty Stress Murine Model. Biomed. Res. Int. 2013, 1-9. doi:10.1155/ 2013/792940

Yu, F., Takahashi, T., Moriya, J., Kawaura, K., Yamakawa, J., Kusaka, K., et al. (2006). Traditional Chinese Medicine and Kampo: a Review from the Distant Past for the Future. J. Int. Med. Res. 34, 231-239. doi:10.1177/ 147323000603400301

Zhao, J., Zhao, L., Zhang, S., and Zhu, C. (2020). Modified Liu-Jun-Zi Decoction Alleviates Visceral Hypersensitivity in Functional Dyspepsia by Regulating EC cell-5HT3r Signaling in Duodenum. J. Ethnopharmacology 250, 112468. doi:10. 1016/j.jep.2019.112468

Conflict of Interest: The authors declare that the research was conducted in the absence of any commercial or financial relationships that could be construed as a potential conflict of interest.

Copyright $\odot 2021$ Inokuchi, Masaoka and Kanai. This is an open-access article distributed under the terms of the Creative Commons Attribution License (CC BY). The use, distribution or reproduction in other forums is permitted, provided the original author(s) and the copyright owner(s) are credited and that the original publication in this journal is cited, in accordance with accepted academic practice. No use, distribution or reproduction is permitted which does not comply with these terms. 